\section{LA IMPLEMENTACIÓN DEL TRIBUTO VERDE, COMO ELEMENTO QUE CONTRIBUYE AL FOMENTO DE LA CULTURA VERDE}

\author{
THE IMPLEMENTATION OF THE GREEN TAX, AS ELEMENT THAT \\ CONTRIBUTES TO THE PROMOTION OF GREEN CULTURE
}

\author{
Génesis Franco, Paola Loyo y Arianny Sánchez
}

Artículo recibido octubre 2019 | Arbitrado noviembre 2019 | Publicado 01 de enero 2020

\section{Resumen}

El presente artículo, producto de un trabajo de investigación que tuvo como objetivo proponer la implementación del Tributo Verde como elemento que contribuye al fomento de la Cultura Verde. La investigación fue orientada por la teoría de cultura verde, compromiso, cuidado ambiental, responsabilidad y también junto a los hechos y cambios positivos que se han generado en otros países por la inclusión del Tributo Verde. Metodológicamente se apoya en una investigación no experimental basada en el análisis de los objetivos específicos, con el fin de indagar sobre los posibles beneficios que obtendría la incorporación del Tributo Verde, enmarcado bajo la modalidad de proyecto factible, con estudio documental y de campo, la información analizada es de primera mano, contando con la participación de los encargados de comercios, elegidos como los protagonistas clave para el logro y cumplimiento del objetivo propuesto, se hicieron visitas a los diferentes locales con el fin de poder recolectar información oportuna para llegar a una conclusión clara y veraz, basada en lo que realmente conocen los comerciantes sobre este modo de recaudación y la finalidad que se logra al incorporar el Tributo Verde.

Palabras clave: Tributo verde, cultura verde, ambiente

\begin{abstract}
This article, the product of a research project that aimed to propose the implementation of the Green Tribute as an element that contributes to the promotion of Green Culture. The research was guided by the theory of green culture, commitment, environmental care, responsibility and also with the positive facts and changes that have been generated in other countries by the inclusion of the Green Tribute. Methodologically it is supported by a non-experimental investigation based on the analysis of the specific objectives, in order to inquire about the possible benefits that the incorporation of the Green Tribute would obtain, framed under the feasible project modality, with documentary and field study, the Information analyzed is first-hand, with the participation of those in charge of shops, chosen as the key protagonists for the achievement and fulfillment of the proposed objective, visits were made to the different premises in order to be able to collect timely information to reach a Clear and truthful conclusion, based on what traders really know about this mode of collection and the purpose that is achieved by incorporating the Green Tribute.
\end{abstract}

\section{Génesis Franco}

abriana_09@hotmail.com Universidad de Carabobo, Venezuela

Licenciada en Contaduría Pública de la Universidad de Carabobo, Venezuela.

\section{Paola Loyo}

paolaloyo77@gmail.com Universidad de Carabobo, Venezuela

Licenciada en Contaduría Pública Universidad de Carabobo, Venezuela. Experiencia en el campo empresarial.

\section{Arianny Sánchez}

anylabella_1@hotmail.com Universidad de Carabobo, Venezuela

Contadora Pública egresada de la Universidad de Carabobo, Venezuela. 


\section{INTRODUCCIÓN}

El desarrollo de la sociedad y de las nuevas tecnologías han sido uno de los factores que conducen el deterioro ambiental, causando así preocupación en el ámbito nacional e internacional, por lo tanto, se han ido implementando distintas políticas ambientales que buscan el aumento de la calidad del medio ambiente, logrando el equilibrio entre el desarrollo sostenible y la naturaleza, a través de medidas regulatorias.

En la actualidad se ha promovido la utilización de tributos con vocación ambiental, los cuales están destinados a cumplir con la prevención y reparación de la contaminación; por esta razón surge lo que se conoce hoy en día como tributo verde.

El Tributo Verde es una de las herramientas más utilizadas en el mundo gracias a que la sociedad cuenta con una cultura verde, dentro de los cuales están algunos países que conforman el continente Europeo entre los que destacan: España, Italia, Dinamarca, Alemania, Francia, Holanda, Austria y Bélgica, donde su aplicación ha generado cambios ventajosos para reducir el deterioro del ambiente, apoyando decididamente la introducción de estos tributos verdes como instrumento para cumplir los compromisos de reducción de emisiones contaminantes asumidos por Europa en el marco del Protocolo de Kioto.

Algunos países de América conformados por Chile, Colombia, Ecuador y México se están iniciando en la utilización de este instrumento a través del marco legal. En Venezuela no existe una cultura verde que origine la implementación de los tributos verdes, pero si se lleva a cabo los derechos ambientales guiados hacia un desarrollo sustentable establecidos en la Constitución de la República Bolivariana de Venezuela, basándose en esta la creación de la Ley del
Ambiente, La Ley Penal del Ambiente, normativas y ordenanzas.

Para efectos de esta investigación, se asume la definición de Impuestos Verdes de Sterner y Rodríguez (2004), quienes señalan que los impuestos verdes o ambientales son instrumentos de política ambiental mediante los cuales pueden influir los impactos ecológicos, disminuyendo o eliminando determinados problemas modificando situaciones y comportamientos perjudiciales para el medio ambiente.

Por lo tanto, la incorporación de este impuesto origina la disminución del deterioro ambiental ocasionado por los diferentes agentes contaminantes, haciendo énfasis en el lema "Quien contamina, paga", promoviendo de esta manera una mejor calidad de vida a la sociedad, llevándolo a cabo principalmente los entes competentes, ya que es una obligación fundamental proteger y mantener el ambiente, y con la activa participación de la sociedad para que las personas se desenvuelvan en un ambiente libre de contaminación.

En este orden de ideas según el autor García (2010) indica claramente que los impuestos verdes también conocidos como impuestos medio ambientales no deben ser simplemente una medida recaudatoria sino que deben de tener claramente un destino para financiar la mejora del medio ambiente. Partiendo de lo antes expresado, los ingresos que se obtengan por medio de este concepto deben estar dirigidos única y exclusivamente a actividades, acciones, tareas y funciones que estén encaminadas para revertir el daño al ecosistema y evitar que continúen actos desmedidos afectando nuestro hábitat por parte de la población, evitando que lo percibido se desvíe del fin por el cual fue implementado. 
Al hablarse de la implementación de los Tributos verdes, se encuentran limitantes que en cierta parte influyen en la no mejora de la conservación ambiental, dando como resultado que en la consecución de los objetivos se presenten retardos 0 simplemente no se logre su fin.

El Estado es uno de los elementos fundamentales para la conservación del ambiente, colocándolos desde la perspectiva de unión entre ellos con el entorno, los cuales ayudan y apoyan a la conservación del mismo. Las diversas estrategias de planificación por parte de las gobernaciones para llevar a cabo la gestión laboral ambiental con resultados eficientes, se distorsionarían y no se encaminarían de acuerdo a lo programado cuando se presentan desaprobaciones o retardos a nivel de financiamiento por parte de autoridades de mayor rango.

Estos cuentan con pocos recursos que le proporciona el estado para subsanar sus compromisos y para llevar a cabo las metas que le competen, los cuales establecen impuestos, tasas y contribuciones para generar ingresos, aunque la mayoría va destinada a actividades que no están relacionadas con la preservación ambiental. Sin embargo, los municipios tratan de subsistir con tan precario presupuesto, no se dan a la tarea de buscar otras posibles fuentes de ingresos para cubrir necesidades, lo que ocasiona que éste sea un tema presente en nuestro día a día, el cual no se le ha dado la gran importancia que tiene.

El trabajo de investigación enunciado en este artículo, quiso dar respuestas a la siguiente interrogante: ¿De qué manera se puede proponer la implementación de Tributos Verdes, como elemento que contribuye al fomento de la cultura verde en el Estado Carabobo, Municipio San Diego? Además, se planteó como objetivo General:
Proponer la implementación de Tributos Verdes, como elemento que contribuye al fomento de la cultura verde en el Estado Carabobo, Municipio San Diego. Y como Objetivo Específicos: Diagnosticar los beneficios que traerá a la sociedad la implementación de Tributos Verdes en el Municipio San Diego; Analizar las ventajas que obtendría la incorporación de Tributos Verdes en materia de recaudación en el Municipio San Diego; Diseñar lineamientos operativos que fundamenten la implementación de Tributos Verdes en el Municipio San Diego.

Es necesario señalar, que aunque el estudio referido se circunscribe a un escenario especifico en la República Bolivariana de Venezuela, se ha querido en este artículo presentar los resultados obtenidos, con la finalidad de que puedan ser referentes para todos los interesados en el tema.

\section{MÉTODO}

La investigación estuvo enmarcada bajo la modalidad de proyecto factible, con estudio documental y de campo; se dio una propuesta para solucionar la problemática que afecta a la comunidad del Municipio San Diego. Por consiguiente, se respondió al problema estudiado, realizando un diagnóstico, debido a que se observó la realidad y la gran exactitud de las distintas variables, sin modificar o manipular los diversos factores que han venido generando el deterioro del ambiente, originando de esta manera que la situación estudiada fuera expuesta tal cual y como se encuentra en la actualidad, es decir, los datos son de primera mano, originales, producto de la investigación en curso, tomados directamente sin intermediación alguna. Fue factible dar respuesta a esta problemática, porque se contó con el apoyo de la alcaldía, 
de la tecnología y otros recursos que le garantizan al municipio beneficios.

Para efectos de investigación, la población fue limitada y estuvo constituida por las pequeñas y medianas entidades domiciliadas en el municipio San Diego la cual estuvo comprendida entre las urbanizaciones de Los Jarales y La Esmeralda, estando relacionadas por una o más características comunes, que son las que inducen a emprender el proceso de estudio de la misma. Todos los miembros de la población tuvieron iguales posibilidades de ser seleccionados como integrantes de la muestra y de esta manera se garantizó obtener los resultados esperados.

En el caso de la muestra, no se eligió al azar, sino que, por razones determinadas, se decido quiénes serían los integrantes de la misma, así se realizó un muestreo no probabilístico intencional. Por consiguiente, la muestra estuvo comprendida únicamente por siete (7) pequeñas y medianas entidades de distintas actividades económicas, tomando en cuenta esta cantidad por falta de recursos, dentro de los cuales se especifican: en tiempo y dinero.

Se utilizó un cuestionario de preguntas cerradas politómicas o categorizadas, como herramienta para conocer y simplificar la información que se obtuvo de la fuente de la investigación. Estuvo formulada por un total de 19 preguntas (ítems), las cuales estuvieron compuestas por cuatros (4) opciones de respuestas (mucho, suficiente, poco y nada).

\section{RESULTADOS Y DISCUSIÓN}

Como resultado del trabajo realizado, surgió la propuesta para la implementación del tributo verde, como elemento que contribuye al fomento de la cultura verde la cual se presenta a continuación.
El tributo verde es una innovación a nivel mundial, cuya función general es el de recaudar dinero, los fondos que se obtendrán tienen como meta principal la prevención del deterioro del ambiente y de ser necesario revertir los daños causados al mismo.

La propuesta consiste en la implementación del Tributo Verde, como elemento que contribuye al fomento de la cultura verde en el municipio San Diego del estado Carabobo; esto como herramienta eficaz de recaudación en pro del cuidado y preservación del ambiente, así como también el poder crear conciencia impulsando una cultura verde dentro del personal de las distintas organizaciones, para poder llevar a cabo la ejecución de medidas tributarias o políticas ambientales para la consecución de los objetivos.

La misma se basa en la determinación de una alícuota impositiva estimada, la cual será aplicada para los sujetos pasivos que realicen actividades económicas en el municipio; para el cálculo del tributo, se tomara como base los ingresos netos obtenidos por los contribuyentes en un ejercicio económico, tomando en cuenta que el monto determinado no debe afectar de manera significativa en la economía de la organización y así poder gravar la actividad productiva del agente contaminante.

El plazo de pago será de tres meses contados a partir de la fecha del ejercicio económico, este se realizará por la página web del municipio, así como se realizan los pagos de los demás impuestos, además de recaudar lo que se espera es influir en la actuación del sujeto pasivo para dirigir su conducta hacia comportamientos deseados y que son beneficiosos para el medio ambiente, incentivando el uso de tecnologías limpias. 
En cuanto a la misión será impulsar en las pequeñas y medianas entidades ubicadas en el municipio San Diego del estado Carabobo una reflexión ambiental y la protección al medio ambiente, mediante la utilización de métodos regulatorios a través de la recaudación del tributo, acorde a la actividad que emplean en su negocio, generándole al personal amplios conocimientos en cuanto a conservación, cuidado y medidas preventivas ambientales se refiere; además otorgándole al comerciante una inducción más extensa del Tributo Verde, proporcionándole al mismo tiempo, los procedimientos a seguir para el cumplimiento de las normativas establecidas.

De igual forma la visión será lograr que las pequeñas y medianas entidades del Municipio San Diego del Estado Carabobo, se comprometan a adoptar el Tributo Verde, como medida preventiva para combatir la contaminación ambiental y que a su vez se lleve a cabo en los distintos municipios del Estado Carabobo, lográndose expandir por todas las regiones del país, originando que su factibilidad como medio de recaudación sea expuesta y aceptada a nivel nacional, pudiéndose solucionar el deterioro ambiental en beneficio de las generaciones futuras.

De acuerdo a la aplicación del instrumento de recolección de datos es factible la aplicación del tributo en el municipio, ya que se cuenta con la receptividad de la población para colaborar con la alcaldía en realizar actividades para revertir el daño al ambiente, además se realizará charlas y talleres para informar a la población sobre esta innovación, se debe trabajar en conjunto para tener éxito, y lograr el objeto que busca implementar el tributo verde.
Ahora bien, es factible implementar el tributo ya que el municipio dentro de su estructura organizacional, cuenta con un departamento de dirección de hacienda encargado de recaudar los tributos, además el municipio posee infraestructura tecnológica, de fácil acceso para el contribuyente, por lo cual se anexa este nuevo tributo a la página web de la alcaldía para realizar el pago.

Se plantea como objetivo general: Recaudar el Tributo para revertir el deterioro del ambiente, desarrollando procedimientos ambientales que permitan la eficacia y receptividad de los comerciantes al incorporarse el Tributo Verde en el municipio San Diego del estado Carabobo. $Y$ como objetivos específicos: Identificar los comercios que incurren en la contaminación ambiental en el Municipio San Diego del estado Carabobo; Determinar el método de recaudación más adecuado para los negocios del Municipio San Diego del estado Carabobo; Reducir los costos adicionales de los comercios que debían erogar al verse afectados por la conducta irracional de los ciudadanos y viceversa en el municipio San Diego del estado Carabobo; Gravar la utilidad neta de los contribuyentes del municipio San Diego del estado Carabobo.

\section{Discusión}

Según el autor Tondini (2005), los tributos verdes son instrumentos fiscales que contribuyen a la protección y defensa del medio ambiente a través de las figuras impositivas con la finalidad de estimular e incentivar comportamientos más respetuosos con el entorno natural, modificando la conducta de productores y consumidores para sustituir productos contaminantes por otro tipo de productos que no causen daños a la ecología. La recaudación que proporciona estos tributos 
compensa el impacto de los recursos naturales que originan las conductas humanas, internalizado el costo que una determinada actividad tiene sobre el medio ambiente.

Por consiguiente, la incorporación de este tipo de modalidad tributaria ayudará a suprimir el daño que se le está causando al ecosistema, disminuyendo el grado de contaminación generado por los distintos factores contaminantes, el cual estaría evidenciado a través de esas diversas recaudaciones que dependerán de la magnitud de deterioro que cause, originando un control en el uso racional de los bienes naturales alcanzando así la conservación, defensa y mejoramiento del medio ambiente como método idóneo para lograr un cambio de actitud del ciudadano con el fin de preservar y optimizar el ambiente.

Tondini (2005), plantea que el índice más utilizado en los tributos ambientales es el consumo debido a que es el único medio a través del cual se puede encontrar relación directa entre una actividad contaminante y una manifestación de capacidad económica, pero no se debe olvidar que su objetivo más que recaudatorio es el incentivar conductas tendentes a la conservación del medio ambiente.

Según la Agencia Europea de Medio Ambiente (AEMA) (2008), de acuerdo a sus investigaciones afirma que: Son instrumentos especialmente eficaces para la internalización de las externalidades, es decir la incorporación directa de los costes de los servicios y perjuicios ambientales (y su reparación) al precio de los bienes, servicios y actividades que los producen; y para contribuir a la aplicación del Principio de Pago por el Contaminante y a la integración de las políticas económica y ambiental.

Asimismo, pueden proporcionar incentivos para que tanto consumidores como productores cambien de comportamiento en la dirección de un uso de recursos más eco-eficiente; para estimular la innovación y los cambios estructurales; para reforzar el cumplimiento de las disposiciones normativas; pueden aumentar la renta fiscal, que puede utilizarse para mejorar el gasto en medio ambiente; y/o para reducir los impuestos sobre el trabajo, el capital y el ahorro; además pueden ser instrumentos de política especialmente eficaces para abordar las prioridades ambientales actuales a partir de fuentes de contaminación tan difusas como las emisiones del transporte (incluido el transporte aéreo y marítimo), los residuos (por ejemplo; envases, baterías) y agentes químicos utilizados en agricultura (p.ej., pesticidas y fertilizantes).

Existen diferentes tipos de tributos verdes, dentro de las cuales tenemos las siguientes: según Alarcón (2002) Tributos sobre emisiones, gravan directamente las descargas contaminantes al medio a través de estimaciones directas de la base imponible. Tienen la particularidad de ser tributos complejos de aplicar por cuanto requieren la experticia de especialistas más allá del Derecho y la Economía para determinar el hecho y la base imponible, por lo tanto, puede resultar costosa esa visión interdisciplinaria; Tributos sobre productos, en este caso se aplica el impuesto utilizando como hecho imponible la producción o el consumo de determinados bienes cuyo uso es peligroso para el medio ambiente. Los que gravan el consumo de determinados bienes de producción, tales como tributos sobre los insumos de los procesos productivos y; los que gravan el consumo de productos acabados o tributos sobre bienes de consumo final.

A raíz de las crisis ecológicas que amenazan la salud del planeta, se da origen a 
la aparición de una Cultura Verde, considerada como una corriente ideológica que apoya el desarrollo sostenible de la economía y la ecología entrelazada por la ciencia y la política. De acuerdo a Pivonia y otros (2010), para hablar de la cultura verde es necesario hablar de la vida, de la realidad tal cual es en la actualidad. Lo que importa en esta cultura es enfocarse en algo que se pueda re-inventar, re- imaginar, re-plantear, y aplicarlo a conciencia; re-educar y revivir nuestra amada civilización está en nosotros. Si queremos mejorar la vida en la Tierra, es decir, formar parte de la cultura consciente, debemos cambiar nuestra forma de pensar y actuar, incluso nuestra forma de leer.

Hoy en día, por los diferentes cambios climáticos se han ido ocasionando inmensas olas de calor en todo el territorio nacional venezolano, esto a su vez está acompañado de un deterioro del medio ambiente, esta situación es consecuencia de una variedad de hechos que se han ido generando al transcurrir de los años, sea por la creciente tasa poblacional, la no adecuada utilización de los diversos recursos naturales, entre los que están la tala y la quema de árboles, un mayor consumo de energía eléctrica puesto que es proveniente de una fuente hidroeléctrica lo que ocasiona una demanda de un recurso renovable como lo es el agua.

Por tal razón, la sociedad tiene que verse involucrada con todos los aspectos fundamentales que vinculen al ambiente, en primordial con lo que se refiere a una cultura verde, ya que este es el nexo principal para el comienzo de una preservación ambiental, de manera que en cada ciudadano exista una consciencia verde la cual ocasiona un manejo adecuado y sostenible de los residuos; en efecto la incorporación del tributo verde tiene que estar estrechamente relacionado con lo que es una cultura verde, para así empezar con una eficiente labor en materia de recaudación ambiental.

Basándose en las respuestas obtenidas en la investigación referida, la mayoría no tiene conocimiento del tributo verde, del impuesto verde y mucho menos de los beneficios que este traería a la sociedad al implementarlo, pero si están de acuerdo a contribuir con el pago del mismo ya que lo asocian como un medida para ayudar a reversar los efectos de la contaminación ambiental que afecta a toda la población del municipio san diego, que a su vez el dinero recaudado será dirigido a realizar acciones por parte de la dirección de hacienda del municipio encargada de recaudar los demás tributos.

Muchos son los beneficios que este tributo traería a la población, entre ellas, actividades que serán dirigidas para revertir el daño causado al ambiente, así como evitar que continúen actos por parte de la población que sigan afectando el habitad; crear interés, responsabilidad para iniciar una cultura verde, concientizar a los ciudadanos sobre otros aportes que pueden hacer para cuidar el ambiente, despertando el sentido de pertenencia, incentivar comportamientos más respetuosos, modificando la conducta del productor, del prestador del servicio, y del mismo consumidor para cambiar productos contaminantes por otros que no causen daño al ambiente.

Llevar a cabo la implementación de este tributo es viable, porque el municipio cuenta con una buena estructura organizacional en materia tributaria, la población está dispuesta en formar parte de esta innovación, a su vez el ingreso por este tributo será destinado únicamente a su fin y podrá financiarse el mismo. 
De acuerdo a las respuestas de cada encuestador realizadas a través del cuestionario, se puede concluir que un número significantes de comerciantes están dispuestos a colaborar en materia de recaudación con respecto al ambiente a través de la implementación del tributo, sin embargo, se pudo notar que existe una falta de información por parte de los comerciantes referente al tema del ambiente, la no motivación en los negocios respecto a la realización de actividades de cómo cuidar y prevenir el deterioro del ambiente esto no es impedimento para poder ser portadores tanto de palabra como de ejecución para el comienzo de este innovador y distinto tributo.

Al llevarse a cabo la propuesta de implementación de este tributo, se producirían mejoras en aquellos sectores que se han visto afectados y deteriorados a causa del no y mal cuidado del entorno. El llevar a los distintos encargados de los comercios la debida información en materia de cuidado o prevención ambiental o también a como minimizar el daño ocasionado al mismo esto reflejaría un gran cambio positivo en el hábitat así como también se incursionaría dentro de los negocios una comunicación entre patrono, encargado y empleados para llevar a cabo una gestión ambiental, esto generaría una incentivación desde adentro hacia afuera es decir, desde las personas que integran el local hacia la comunidad.

En conclusión, con base a las respuestas obtenidas por los encuestados, la mayoría no tiene conocimiento acerca las leyes, normas y reglamentos establecidos en materia ambiental, por ello es necesario que el Departamento de Hacienda del municipio desarrolle estrategias para que los negocios manejen por lo menos los principales artículos básicos en materia ambiental y conozcan de las sanciones en la que puede incurrir su establecimiento por no cumplir con las leyes.

\section{CONCLUSIONES}

Implementar el tributo verde traerá beneficios a la sociedad, gracias a este se podrán reducir enfermedades para tener mejor calidad de vida como lo establecen las leyes en materia ambiental, las enfermedades causadas por la contaminación al ambiente, ya sea por la basura, quema de desechos orgánicos e inorgánicos, que afectan la salud de las personas y su bienestar. Otro beneficio consistiría en contribuir a concientizar a la población en la relación que existe entre el hombre y el medio ambiente para así conseguir incentivar valores y aptitudes que aporten de manera favorable a cuidar el medio ambiente.

Las ventajas de incorporar el tributo verde, para el municipio, consiste en obtener una fuente de ingreso para financiar actividades, proyectos, talleres e instituciones enfocadas en la protección del medio ambiente, sin tener que destinar parte de los ingresos que le concede el ejecutivo para administrar el municipio, lo que significa más renta para invertir, en servicios públicos, alumbrado, seguridad y otras funciones del alcalde. La ventaja para los productores, comerciantes, prestador de servicio, consumidor, serian que disminuiría el dinero que deberían pagar, al verse afectados por utilizar recursos contaminantes, que afecten a otros. Igualmente, aumenta el capital de los habitantes del municipio y el ahorro.

Los lineamientos operativos que fundamenten la implementación del tributo verde, en primer lugar, realizar actividades, charlas, hacer propaganda para que sea aceptado este tributo, ya que existe una 
minoría que no está de acuerdo en colaborar, luego detectar aquellas actividades que tengan un mayor impacto en el ambiente, también crear una ley de este tributo que lo regule.

Lo primero que se recomienda es capacitar al personal de la alcaldía de san diego, con cursos en materia tributaria, para que brinden correcta información a los comercios, y la población en general, ya que estos deben tener conocimiento para poder ejercer el puesto; otra recomendación seria realizar campañas educativas para promocionar el tributo verde, comentando a quienes beneficia, que se busca con esta innovación, que se hará con el dinero recaudado, las multas, quienes deben pagar, en fin dar a conocer el tributo para conseguir la receptividad de la población, las charlas se podrían realizar desde los colegios para comenzar a sembrar una cultura verde desde niños, hasta el puesto de trabajo. También podrían mejorar y acondicionar la estructura física de la dirección de hacienda, para que sea eficiente y se realice con rapidez la declaración pago de tributo.

\section{REFERENCIAS}

AEMA (2008). El Tributo Ambiental. Aplicación y efectividad sobre el medio ambiente Recuperado de http://reports.eea.eu.int/92-9167-0006sum/es/page001.html [consulta 3 febrero 2004]

Alarcón; G. (2002). La fiscalidad ambiental. El fenómeno tributario y su relación con el medio ambiente" en Justicia ecológica y protección del medio ambiente. Coordinado por Teresa Vicente Giménez, Editorial Trotta.

García, J. (2010). Los Impuestos Verdes: sus fundamentos y tendencias en el mundo. Recuperado de http://www.finanzasytributos.com/main /mdoc_944_impuestos-verdesfundamentos-tendencias-mundo.html. [Consulta: 26-06-2010]

Pivonia, S; Gerstl, Z; Maduel, A; Levita, R; (2010). Management of Monosporascus cannonballus sudden wilt of melon by soil application of fungicides. European Journal Plant Pathology 128: 201-209 